\title{
Reliable identification of prostate cancer using mass spectrometry metabolomic imaging in needle core biopsies
}

\author{
Nicole Morse ${ }^{1,2} \cdot$ Tamara Jamaspishvili,2 $^{1, \text { David Simon }^{3}} \cdot$ Palak G. Patel $^{1,2} \cdot$ Kevin Yi Mi Ren $^{2} \cdot$ Jenny Wang ${ }^{1,2} \cdot$ \\ Richard Oleschuk $^{3} \cdot$ Martin Kaufmann ${ }^{4,5} \cdot$ Robert J. Gooding ${ }^{1,2,6} \cdot$ David M. Berman $\mathbb{( I}^{1,2}$
}

Received: 17 October 2018 / Revised: 6 April 2019 / Accepted: 29 April 2019 / Published online: 3 June 2019

(c) United States \& Canadian Academy of Pathology 2019

\begin{abstract}
Metabolomic profiling can aid in understanding crucial biological processes in cancer development and progression and can also yield diagnostic biomarkers. Desorption electrospray ionization coupled to mass spectrometry imaging (DESI-MSI) has been proposed as a potential adjunct to diagnostic surgical pathology, particularly for prostate cancer. However, due to low resolution sampling, small numbers of mass spectra, and little validation, published studies have yet to test whether this method is sufficiently robust to merit clinical translation. We used over 900 spatially resolved DESI-MSI spectra to establish an accurate, high-resolution metabolic profile of prostate cancer. We identified 25 differentially abundant metabolites, with cancer tissue showing increased fatty acids (FAs) and phospholipids, along with utilization of the Krebs cycle, and benign tissue showing increased levels of lyso-phosphatidylethanolamine (PE). Additionally, we identified, for the first time, two lyso-PEs with abundance that decreased with cancer grade and two phosphatidylcholines (PChs) with increased abundance with increasing cancer grade. Importantly, we developed and internally validated a multivariate metabolomic classifier for prostate cancer using 534 spatial regions of interest (ROIs) in the training cohort and 430 ROIs in the test cohort. With excellent statistical power, the training cohort achieved a balanced accuracy of $97 \%$ and validation on testing data set demonstrated $85 \%$ balanced accuracy. Given the validated accuracy of this classifier and the correlation of differentially abundant metabolites with established patterns of prostate cancer cell metabolism, we conclude that DESI-MSI is an effective tool for characterizing prostate cancer metabolism with the potential for clinical translation.
\end{abstract}

Supplementary information The online version of this article (https:// doi.org/10.1038/s41374-019-0265-2) contains supplementary material, which is available to authorized users.

David M. Berman

bermand@queensu.ca

1 Cancer Biology \& Genetics, Queen's Cancer Research Institute, Queen's University, Kingston, ON K7L 3N6, Canada

2 Department of Pathology \& Molecular Medicine, Queen's University, Kingston, ON K7L 3N6, Canada

3 Department of Chemistry, Queen's University, Kingston, ON K7L 3N6, Canada

4 Department of Surgery, Queen's University, Kingston, ON K7L 3N6, Canada

5 Department of Biomedical and Molecular Sciences, Queen's University, Kingston, ON K7L 3N6, Canada

6 Department of Physics, Engineering Physics \& Astronomy, Queen's University, Kingston, ON K7L 3N6, Canada

\section{Introduction}

The recent emergence of the field of metabolomics and advances in related assay technologies have opened new avenues for cancer research and clinical applications in pathology laboratories and in operating theaters. Metabolomic profiling has the potential to supplement existing profiling methods by more rapidly and inexpensively identifying and characterizing disease states, including cancer [1].

Typically, metabolomic research is performed using nuclear magnetic resonance (NMR) spectroscopy or mass spectrometry (MS) techniques to investigate amino acids, sugars, nucleic acids, FAs, lipids, steroids, and proteins within a biological sample. NMR can identify the molecular structure of a metabolite; however, it lacks sufficient sensitivity to detect low abundance analytes. Untargeted mass spectrometry (MS) methods typically first separate metabolites through liquid chromatography (LC) or gas chromatography (GS) systems [2-4]. MS methods are more sensitive meaning that they can detect low abundance 
metabolites and can detect many different molecular species concurrently. Both methods require tissue homogenization and extensive sample preparation, therefore spatial distribution of the ions is lost, making it difficult to study variations in metabolite abundance arising from tissue heterogeneity [5].

Combining metabolomic techniques with imaging capabilities is a useful way to study the metabolic profile of different regions within a tissue. Desorption electrospray ionization mass spectrometry imaging (DESI-MSI) is an ambient ionization technique that can analyze sugars, FA and lipids directly from the tissue surface [5]. With minimal sample preparation, DESI can analyze a metabolic profile at a resolution of $50 \mu \mathrm{m}$. Spatial coordinates and ion intensities are combined to re-create the image of the tissue [5]. Ion heatmaps are then matched with pathologically validated photomicrographs of hematoxylin and eosin stained (H\&E) histologic sections to identify regions of pathological interest and the corresponding metabolic profile [6]. This technology also has drawbacks. The lack of normalization techniques and lack of standardized analytic protocols have posed challenges that raise questions regarding its reliability and potential clinical utility.

Prostate cancer has been a focus of study using this technique. Early stage prostate cancer is difficult to detect by palpation or imaging, posing a challenge to prescribing appropriate therapeutic and surgical intervention [7]. Unlike colon cancer [8], prostate cancer initiation and progression cannot be subdivided into an orderly sequence punctuated by specific genomic events [9]. Additionally, prostate cancer forms in small cell groupings, therefore requiring techniques with adequate resolution to distinguish cancer cells from surrounding stroma or benign tissue [10]. Thus, few biomarkers reliably distinguish prostate cancers from benign prostate epithelia or indolent prostate cancers from their aggressive counterparts [11]. The distinctive metabolic profiles of prostate epithelia and prostate cancer cells provide opportunities for additional insights into prostate cancer biology, along with new diagnostic and prognostic biomarkers.

As described by Otto Warburg, most cancer cells shut down the mitochondrial Krebs cycle, favoring aerobic glycolysis, a less efficient route to energy production [12]. Prostate cancer, in contrast, shows a more energy efficient metabolic profile and enhanced utilization of the Krebs cycle compared to benign prostate epithelia [13, 14]. In concert with enhanced energy efficiency, prostate cancer cells upregulate de novo FA and lipid synthesis $[15,16]$. These products support cancer cell proliferation in a variety of ways, serving as building blocks for cell membranes, signaling molecules for signal transduction pathways, post-translational modifications of proteins, and fuel [16]. Recent work has also established metabolic signatures that correlate with genetic drivers of the disease. Through use of cell lines, mouse models and human tissue samples, Priolo et al. concluded that prostate cancers driven by the activation of the serine/threonine specific protein kinase $\mathrm{B}$, known as AKT, displayed higher levels of aerobic glycolysis compared to cancers driven by activation of the MYC proto-oncogene, which displayed dysregulated lipid metabolism [2].

To date, two groups have established metabolic profiles associated with prostate cancer using DESI-MSI. No previous study has analyzed more than 28 regions of interest (ROIs) in cancer tissue. Kerian et al. utilized 100 biopsy samples (26 of which were cancer) consisting of merged ROIs to develop a principal component analysis (PCA)/linear discriminant analysis (LDA) classifier composed of $\mathrm{PE}, \mathrm{PCh}$, phosphatidylserine (PS) and phosphatidylinositol (PI) species that achieved $98 \%$ cross validation accuracy with the training cohort but was not validated with a separate cohort [17]. Using larger tissue sections from surgically removed prostates, Banerjee et al. developed a LASSO model from 28 merged cancer ROIs [18]. This model was then validated on 18 merged ROIs from a separate cohort, demonstrating $94 \%$ agreement. Both studies performed classification on a per sample base, therefore all ROIs within a sample were averaged to obtain a single classification. This low resolution approach limits statistical power as well as limiting information regarding heterogeneity within a sample. For example, the classifiers from these studies do not demonstrate sufficient validation and/or spatial resolution to be useful in intraoperative surgical guidance.

In this study, we have developed an analytical workflow to address these problems through accurate assessment of DESI metabolomic profiles to identify, at $150 \mu \mathrm{m}$ spatial resolution, differences between benign and cancer tissue. We analyzed prostate needle biopsy tissue and obtained multiple 9-pixel ROIs $(\sim 150 \mu \mathrm{m} \times 150 \mu \mathrm{m})$ per sample. We conducted statistical analysis using 964 ROIs (675 cancer, and 289 benign), comprising the largest cohort of prostate data points studied with this method to date. In doing so, we built tissue heterogeneity into these models, further improving the confidence of the resulting classifiers and fully utilizing the capabilities of DESI-MSI. Future applications of this approach might include use as ancillary diagnostic tools in the pathology laboratory or real time tissue monitoring to provide intraoperative guidance to surgeons assessing surgical margins.

\section{Material and methods}

\section{Sample selection and collection}

With approval of the Queen's University Research Ethics Board, fresh prostate tissue was collected at Kingston General 
Hospital between August 2016 - July 2017. Following a radical prostatectomy or cystoprostatectomy procedure, the prostate gland was sampled using an 18-gauge needle biopsy gun (CR Bard, Murray Hill, NJ, USA) at 10 pre-determined locations (Supplementary Fig. 1), simulating the template used clinically in transrectal diagnostic biopsies. Selected cores were frozen in optimal cutting temperature compound (OCT, Tissue-Tek, VWR, Radnor, PA, USA), cryosectioned at $12 \mu \mathrm{m}$ thickness, mounted on neutral microscope slides (UltiDent Scientific. St. Laurent, QC, CA) and stored at $-80^{\circ} \mathrm{C}$ for up to 3 months prior to DESI analysis. Hematoxylin and eosin (H\&E) stained slides from biopsy frozen sections and radical prostatectomies (routinely processed) were reviewed by two genitourinary pathologists (TJ and DMB). Grade groups (GGs) were assigned as described [10].

\section{Tissue microarray construction}

Tumor and benign tissue from each case was harvested from the same location as the needle biopsy used for DESIMSI analysis. Cancer and benign tissue were each represented on the tissue microarray (TMA) by $0.6 \mathrm{~mm}$ triplicate cores.

\section{Immunohistochemistry}

Immunohistochemistry (IHC) was performed on $5-\mu m$-thick TMA sections on the automated staining platform (BONDIII, Leica Biosystems, Buffalo Grove, IL, USA) using Ki67 Bond $^{\mathrm{TM}}$ Ready-To-Use Primary Antibody (catalog \#: PA0118, clone ID: MM1, Leica Biosystems, Buffalo Grove, IL, USA) in combination with Bond Polymer Refine Detection kit (Leica Biosystems, Buffalo Grove, IL, USA).

\section{Digital quantification}

Tissue microarrays were scanned at $20 \times$ magnification (Aperio, Leica Microsystems, Buffalo Grove, IL, USA) and uploaded at Aperio ImageScope (Leica Biosystems Imaging, Buffalo Grove, IL, USA). Benign and cancer areas of interest were circled (annotated) on the digital image by a pathologist followed by automated image analysis (HALO, Indica Labs, Corrales, NM, USA). Cores with $<300$ cells of interest (benign epithelial or cancer) were excluded from the analysis. Brown nuclear epithelial staining above background was considered positive. Ki67 proliferation index was calculated by dividing the number of Ki67 positive cells in all cores/per case by the total number of counted cells in the case.

\section{DESI-MSI}

All samples were analyzed using a commercial 2D DESI source (Prosolia, Zionsville, IN, USA) in negative ion mode. Full scan mass spectra were acquired in the mass range $\mathrm{m} / \mathrm{z}$ 50-1100 using a Xevo-G2 XS Q-Tof mass spectrometer (Waters, Milford, MA, USA). Solvent (95\% methanol, $5 \%$ water and $50 \mathrm{pg} / \mu \mathrm{L}$ leucine enkephalin) was infused at a rate of $1.5 \mu \mathrm{L} / \mathrm{min}$. The DESI source was set to the following parameters: capillary temperature $100^{\circ} \mathrm{C}$, capillary voltage $4 \mathrm{kV}$, heated ion transfer tube $500^{\circ} \mathrm{C}$, cone voltage $5 \mathrm{kV}$, collision energy $4 \mathrm{eV}$, capillary incident angle $75^{\circ}$, spray to surface distance $\sim 0.5 \mathrm{~cm}$, sample to inlet distance $\sim 1 \mathrm{~cm}$, and nitrogen gas at $0.6 \mathrm{MPa}$. DESI resolution was set to a pixel size of $50 \times 50 \mu \mathrm{m}^{2}$ with a scan rate of $175 \mu \mathrm{m} / \mathrm{sec}$. After DESI analysis, microscope slides were H\&E stained. Photomicrographs were obtained by scanning the H\&E image at $100 \mathrm{X}$ to allow for the electronic, pathologically validated image to be compared to the DESI images.

Imaging and mass spectral data were mass corrected and normalized to total ion current using HDImaging 1.4 software (Waters, Milford, MA, USA). Ion images were compared with H\&E images to select regions of interest (ROIs) representing either cancer or benign glands, where each ROI consisted of nine $50 \times 50 \mu \mathrm{m}$ pixels of data. The data from each pixel within a ROI were summed, all ROIs from an individual sample were total ion current (TIC) normalized using the formula (Peak intensity of ROI/TIC sum intensity) 10,000 , and were exported for further analysis (Supplementary Table 1).

A random selection of five representative samples $(\sim 10 \%$ of the total cohort) was used to identify a list of abundant tissue ions. This list defined the ions that were used for analysis in all subsequent samples. A $m / z$ tolerance \pm 0.03 was assigned to account for slight variations in measured $\mathrm{m} / \mathrm{z}$ values. After removing ions with median intensities of zero, a final list of 289 ions was generated. Individual ROIs with a median zero intensity for all 289 ions were removed. Therefore, statistical analysis and model building were performed using these 289 ions on a total of 35 samples across 18 patients and 675 cancer ROIs and 289 benign ROIs (Supplementary Table 1).

\section{Ion identification}

Chemical identities were assigned to accurate DESI-MS $\mathrm{m} / \mathrm{z}$ ratio by searching publicly available lipid databases and publications or by comparing to LC-MS/MS-based fragmentation patterns. The $m / z$ of each ion identified by DESI-MS was corrected using the internal mass standard Leu-Enk $(\mathrm{m} / z=554.2615)$. Accurate masses of the differentially abundant ions were matched with metabolites in the Metlin database [19], using a $m / z$ tolerance of \pm 30 parts per million (ppm). Metabolites were further investigated using LC-MS/MS if they remained unmatched after Metlin search or had multiple species with the same 
$\mathrm{m} / \mathrm{z}$ ratio. Tissue lysates were prepared from three representative cases of prostate cancer, GG 2,3, and 5, and subjected to metabolite identification using highresolution LC-MS/MS. Two simple methanol extraction methods [20] and the Bligh and Dyer method [21] were employed to extract lipids. The protocol containing LCMS/MS settings and data processing is provided in Supplemental Material. All precursor ions and associated ion fragments were imported into LipidBlast [22] to match the accurate $\mathrm{m} / \mathrm{z}$ of the precursor and the fragmentation pattern. Chemical identity was confirmed if it was within a 10 -ppm tolerance and had a $\geq 50 \%$ probability based on the fragmentation pattern.

\section{Statistical Workflow}

\section{Identifying differentially abundant ions}

Differentially abundant metabolites had dissimilar variances across cohorts and were often found to be nonnormally distributed. Therefore, we relied on the nonparametric Mann-Whitney $U$ test to establish statistically significant abundances between cancer and benign ROIs. The $p$ values were corrected for false discovery using a Benjamini-Hochburg adjustment [23]. A $\log 2$ fold change between cancer and benign was calculated for each ion using the median intensity of each peak with $q \leq 0.05$. From the resulting list, ions that had an $\mid \log 2$ fold change $\mid \geq 1$ were considered differentially abundant. This procedure was repeated to identify metabolites that were differentially abundant between GG2 and GG $>2$. GG1 ROIs were excluded due to limited sample size (24 ROIs).

\section{Model construction}

Univariate models were generated using the entire dataset. A receiver operating characteristic (ROC) curve with corresponding area under the curve (AUC) was calculated for each of the 25 differentially abundant metabolites.

A train/test internal validation approach was utilized for the remainder of the model building, with half of the prostate cancer cases studied assorted into each of the train and test cohorts. Cases were divided to contain similar breakdowns of GS and percentage of benign and cancer ROIs (Supplementary Table 1). Each cohort contained 9 cases, with the training cohort comprising 534 ROIs (373 cancer and 161 benign) and the test cohort comprising 430 ROIs (302 cancer and 128 benign). The training data was used to construct a logistic regression model. Best selection over 5 metabolites was performed in $\mathrm{R}$ using the leaps package. Forward selection was performed to add single metabolites, one at a time, to the logistic regression model. Varying numbers of metabolites were used to build the model, and training and validation accuracies were evaluated. To explore a more complex PCA/LDA model, PCA was initially performed on the train data using the prcomp function within the stats R package, which also centered and scaled the data. Within a PC, each ion was assigned a weighting value which was used to calculate a single numerical score for each ROI, calculated as the sum of each metabolite's intensity value, subtracted from the mean intensity value across all ROIs, divided by the standard deviation, and multiplied by the weighting value. We selected the PCs and the respective scores for each ROI that explained a cumulative $85 \%$ of the total variance. PCs were then employed as variables used for LDA. LDA was computed using the MASS package in R. LDA was used to build a model composed of PCs optimized to classify benign vs. cancer.

For both logistic regression and PCA/LDA models, a ROC curve was calculated to display the model's AUC and to select a cut-off threshold. The point on the ROC curve farthest away from the diagonal was selected as the initial cut-off value. Using these cut-off values and either the logistic regression or the LDA function, the chosen model's accuracy was assessed using a confusion matrix on the test cohort and the remaining 302 cancer ROIs and 128 benign ROIs.

\section{Results}

\section{Metabolites that distinguish cancer from benign}

We performed univariate differential abundance analysis employing a Mann-Whitney $U$ test for all 289 metabolites (see Methods) and found $p$ and Benjamini-Hochbergcorrected $q$ values for these metabolites. Metabolites that passed a significance cutoff of $q \leq 0.05$ and showed an absolute log-2 fold change of $>1$ were examined. Twelve metabolites were removed because their median values fell within $50 \%$ of the intensity values in the other class. Of the remaining significant metabolites, 14 were more abundant in benign tissue, and 11 more abundant in cancer. Select identification patterns of the metabolites that had a confirmed identity using MS/MS can be seen in Supplementary Figs. 2-5. Many of the significant species are members of the Krebs cycle or intermediates in FA and lipid metabolism. The 14 metabolites that were most abundant in benign tissue consisted mainly of lysoPE species, two PI species and citrate, whereas FAs, PE, $\mathrm{PCh}, \mathrm{PI}$ species and glutamate were amongst the more abundant species in cancer tissue (Table 1). Since many of these differentially abundant metabolites are known 
Table 1 Metabolites with differential abundance between prostate cancer and benign prostate

\begin{tabular}{|c|c|c|c|}
\hline DESI $m / z$ & Theoretical $\mathrm{m} / \mathrm{z}$ & Identity $^{\mathrm{a}}$ & $\begin{array}{l}\log 2 \text { (cancer/ } \\
\text { benign) }\end{array}$ \\
\hline 452.2755 & 452.2783 & LysoPE (16:0) & -2.4 \\
\hline 191.018 & 191.0197 & Citrate/isocitrate & -2.1 \\
\hline 478.2908 & 478.2939 & LysoPE (18:1) & -2.1 \\
\hline 479.2899 & 479.2939 & LysoPE $(18: 1)^{b}$ & -1.9 \\
\hline 480.3053 & 480.3096 & LysoPE (18:0) & -1.9 \\
\hline 481.3072 & 481.3096 & LysoPE $(18: 0)^{\mathrm{b}}$ & -1.6 \\
\hline 367.1028 & Unknown & Unknown & -1.4 \\
\hline 453.2819 & 453.2783 & LysoPE $(16: 0)^{\mathrm{b}}$ & -1.4 \\
\hline 462.2956 & 462.2984 & LysoPE (18:0) & -1.4 \\
\hline 482.3108 & 482.3096 & LysoPE $(18: 0)^{c}$ & -1.4 \\
\hline 867.5124 & 867.5029 & PI (37:6) & -1.4 \\
\hline 454.2851 & 454.2783 & LysoPE $(16: 0)^{\mathrm{c}}$ & -1 \\
\hline 466.3048 & 466.3044 & Cholesterol sulfate ${ }^{\mathrm{b}}$ & -1 \\
\hline 821.5258 & 821.5186 & PI $(33: 1)$ & -1 \\
\hline 146.0443 & 146.0459 & Glutamate & 1.1 \\
\hline 903.5444 & Unknown & Unknown & 7.9 \\
\hline 875.56 & 875.5655 & PI (37:2) & 8.3 \\
\hline 175.023 & 175.0248 & Ascorbic acid & 9.8 \\
\hline 307.2607 & 307.2643 & FA20:2 & 9.9 \\
\hline 799.6622 & 799.6382 & $\begin{array}{l}\text { PCh }(0-38: 2), \text { PCh } \\
(\mathrm{P}-38: 1)^{\mathrm{b}}\end{array}$ & 10 \\
\hline 844.6049 & 844.5862 & PE (44:7) & 10 \\
\hline 826.6789 & 826.6695 & $\begin{array}{l}\text { PCh }(\mathrm{O}-40: 2), \mathrm{PC} \\
(\mathrm{P}-40: 1)\end{array}$ & 10.1 \\
\hline 331.2618 & 331.2643 & FA22:4 & 10.3 \\
\hline 714.5208 & 714.5079 & PE (34:2) & 10.5 \\
\hline 798.6513 & 798.6382 & $\begin{array}{l}\text { PCh }(0-38: 2), \text { PCh } \\
(\mathrm{P}-38: 1)\end{array}$ & 10.5 \\
\hline
\end{tabular}

Metabolites were defined as: FA, PE, PI, PCh with some showing loss of $\mathrm{O}$ or $\mathrm{P}$

FA Fatty acid, PE Phosphatidylethanolamine, PI Phosphatidylinositol, $P C h$ Phosphatidylcholine, $O$ Oxygen, $P$ Phosphorus

${ }^{\text {a }}$ Chemical identity of ions deduced from accurate mass obtained by DESI and LC-MS orbitrap or fragmentation patterns observed during LC-MS/MS analysis

${ }^{\mathrm{b}}$ Represents the $\mathrm{C} 13$ isotope of the parent metabolite

${ }^{c}$ Represents the isotope with two $\mathrm{C} 13$ molecules compared with the parent metabolite

constituents of cell membranes associated with proliferation, we evaluated the Ki67 immunohistochemistry score of adjacent tissue from the same case. This analysis confirmed significantly elevated proliferation rates in cancer cells compared to benign epithelium ( $p=1.6 \mathrm{e}-07)$ and a trend towards increased proliferation with increased cancer grade (Fig. 1). We validated differential abundance of selected metabolites by overlaying heatmaps of ion abundance over photomicrographs of prostate needle

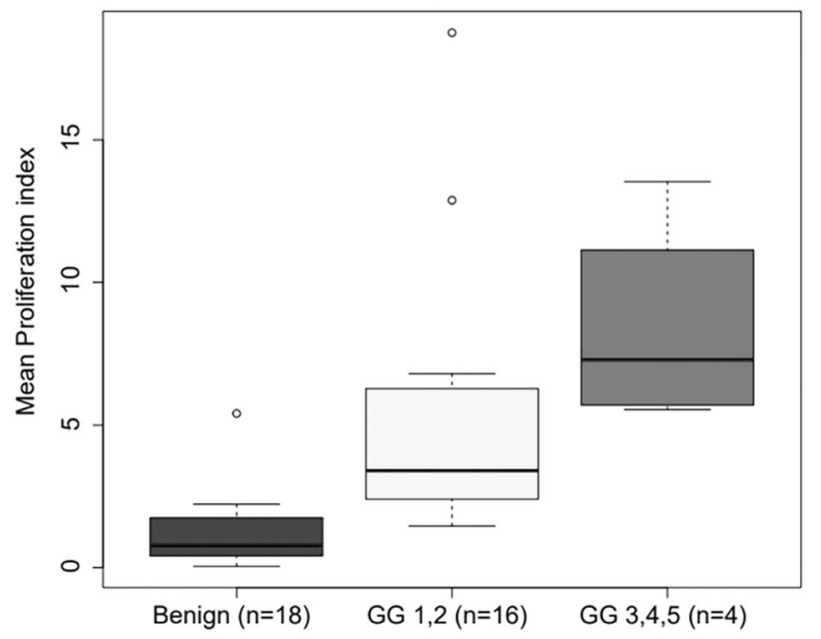

Fig. 1 Proliferation rates in prostate cancer cases profiled by DESI-MSI. Proliferation rate of cells increases from benign to cancer ( $p=1.6 \mathrm{e}-07$, Mann-Whitney $U$ test $)$ and from low/intermediate grade cancer to high grade cancer (trend). Graph shows mean proliferation rate (positive cells/total cells) per case calculated by Ki67 immunohistochemistry and automated digital scoring shows increased proliferation rate in cancer compared to benign cells. Ki67 labeling analysis and DESI-MSI were performed on the same cases. For this and subsequent figures, the colored box depicts $50 \%$ of the intensity value data. Heavy line = median, whiskers show the lesser of the furthest value or 1.5 times the inter-quartile range in each direction, and the open circles are outlier data

cores and comparing them to photomicrographs annotated for cancer and benign regions (Fig. 2).

Univariate cancer/benign classifiers generated individually for each of the 25 differentially abundant metabolites yielded ROC curves with AUCs as large as 0.865 , as observed with lyso-PE(16:0). However, no single metabolite accurately distinguished cancer from benign, as evidence by high rates of false positive and/or false negatives therefore multivariate classifiers were investigated next for their ability to more accurately distinguish cancer from benign (data not shown).

\section{Metabolites that distinguish Grade Groups}

A secondary goal of this work was to assess the ability of DESI-MS imaging of needle core biopsies to identify metabolic features that distinguish more aggressive highergrade prostate cancers from their more indolent grade counterparts. Most prostate cancers form discrete ovoid gland-like structures that are recognized as low grade and assigned Gleason pattern 3. Many of these cancers also contain higher-grade structures in which gland-like structures are fused together or poorly formed, and designated as Gleason pattern 4. The relative contribution of Gleason patterns 3 and 4 drive prognosis, and are reported in a Gleason sum (GS), such that the more prevalent pattern comes first, and the next most prevalent second. For 

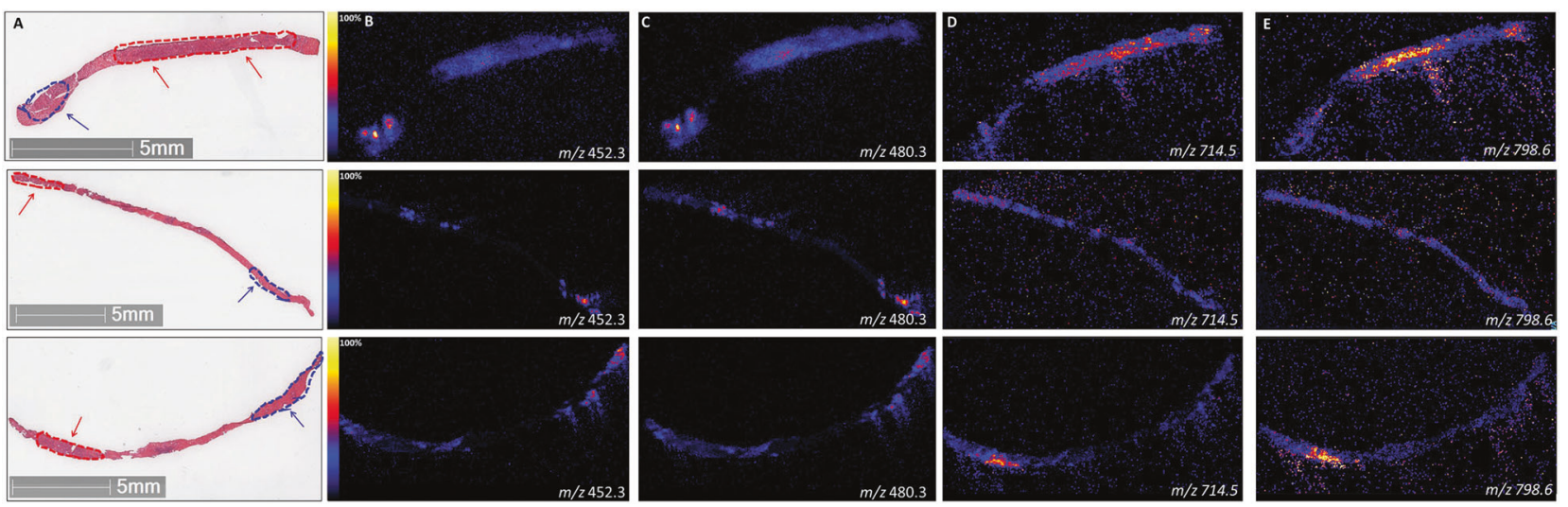

Fig. 2 Spatial distribution of selected ions in prostate cancer cases profiled by DESI-MSI. DESI heatmap images show differential abundance of selected ions that correspond to malignant vs. benign histology on corresponding $\mathrm{H} \& \mathrm{E}$ stained photomicrographs. Prostate needle cores shown are representative across three different patients. a Annotated H\&E stained slides used for comparison to DESI-MSI. Cancer regions are outlined in red and indicated by red arrows. Blue outline and arrows depict regions of benign prostate tissue.

example, pure pattern 3 cancers are reported as GS $3+3=$ 6 and have almost no capacity to metastasize [24]. GS3 + $4=7$ has more pattern 3 and 4 and is less likely to metastasize than GS $4+3=7$, in which pattern four predominates [25]. The newer GG designation for prostate cancer uses a simpler numbering system from 1 to 5 that better reflects risk of disease progression [25]. Our patient cohort was best suited to compare GG2 (Gleason $3+4=7$ ) with GG3 and higher (Gleason $>4+3=7$ ) cases. Despite having the same Gleason sum, GG3 reflects a significantly higher propensity to metastasize as illustrated by a $52 \%$ chance of treatment failure at 5 years [25] GG2 vs. GG3 comparisons are shown for four metabolites. In each case, there is a clear separation between the medians (and means) of metabolites for each grouping of ROIs. Two of these metabolites, lyso-PE (16:0) and lyso-PE (18:0), were more abundant in benign tissue and showed decreasing abundance with increasing cancer grade (Fig. 3). The other two metabolites, $\mathrm{PCh}(0-38: 2) / \mathrm{PCh}(\mathrm{P}-38: 1)$ and $\mathrm{PCh}(\mathrm{O}-40: 2) /$ $\mathrm{PCh}(\mathrm{P}-40: 1)$, showed low abundance in benign tissue and gradually increased with cancer grade (Fig. 3). These results indicated that DESI-MSI can identify metabolites that are associated with aggressive prostate cancer growth and spread.

\section{Multivariate classifier: train/test validation}

We set out to find, using a train/test validation, a classifier that could be validated as highly sensitive and specific for cancer. While no individual metabolite met these standards, we investigated the use of multivariate classifiers. Our analysis explored several multivariate classifiers, including b, c Heatmap image for $m / z=452.3$ [Lyso-PE(16:0)] and $m / z=480.3$ [Lyso-PE(18:0)] respectively. Yellow/red colored-regions match benign regions on the corresponding $\mathrm{H} \& \mathrm{E}$ photomicrographs in a. d, e Heatmap image for $\mathrm{m} / \mathrm{z}=714.5[\mathrm{PE}(34: 2)]$ and $\mathrm{m} / \mathrm{z}=798.6$ respectively. Yellow/red colored-regions match benign regions on the corresponding H\&E photomicrographs in a. Hematoxylin and Eosin (H\&E), Oxygen (o), Phosphorus (p), Phosphatidylcholine (PCh), and Phosphatidylethanolamine (PE) logistic regression and PCA/LDA modeling. We employed the train/test validation approach utilizing 373/161 cancer/ benign ROIs in the training set, and 302/128 ROIs in the test (validation) set. Logistic regression, the simplest multivariate model yielded a 6-metabolite logistic regression model with an AUC of 0.994. Choosing the model's threshold leads to impressive values of sensitivity, specificity and balanced accuracy all being $96 \%$. However, while the test set's sensitivity was reduced to only $89 \%$, its specificity was dramatically reduced to $52 \%$, leading to a balanced accuracy of $78 \%$. To further refine the classifier, we employed unsupervised PCA/LDA. PCA/LDA is an approach used by prior DESI-MSI studies, which, compared to logistic regression, considers a greater proportion of the training data [6]. Several previous studies have demonstrated that models of this type perform well in metabolomic studies [17, 26-29]. We chose to utilize the first 40 PCs, which explained $\geq 85 \%$ of the total variance. These PCs and their associated scores were selected and used in LDA for classifier construction. The supervised LDA model was computed using cancer and benign as the reference classes, and was found to have an AUC of 0.998 on the training set (Fig. 4a). The ROC curve was used to select the final threshold of 0.84 , also shown in Fig. 4a. With this threshold, the LDA model demonstrated a $97 \%$ balanced accuracy, $97 \%$ sensitivity and $97 \%$ specificity for the training cohort, as seen in (Table 2). For the test cohort, the LDA model and the cutoff of 0.84 displayed a balanced accuracy of $85 \%$, sensitivity of $91 \%$, and specificity of $70 \%$ (Table 2). The scoring function for both cohorts, including the model threshold, is shown in Fig. 4b. These results demonstrate that the more complex PCA/LDA model 
Fig. 3 Metabolites that differentiate benign prostate from GG2 and GG3 cancer. Four metabolites demonstrated TIC normalized intensities that were significantly different $(p<0.05$ and $\mid \log 2$ fold change $\mid \geq 1$ ) between benign (blue), GG2 (yellow) and GG3 (red). a $m / z=452.3[$ Lyso-PE (16:0)], b $m / z=480.3$ [Lyso-PE (18:0)], c $m / z=798.64 \mathrm{PCh}$ $(\mathrm{O}-38: 2) / \mathrm{PCh}(\mathrm{P}-38: 1)$, and $\mathbf{d}$ $m / z=826.67 \mathrm{PCh}(\mathrm{O}-40: 2) / \mathrm{PCh}$ (P-40:1)
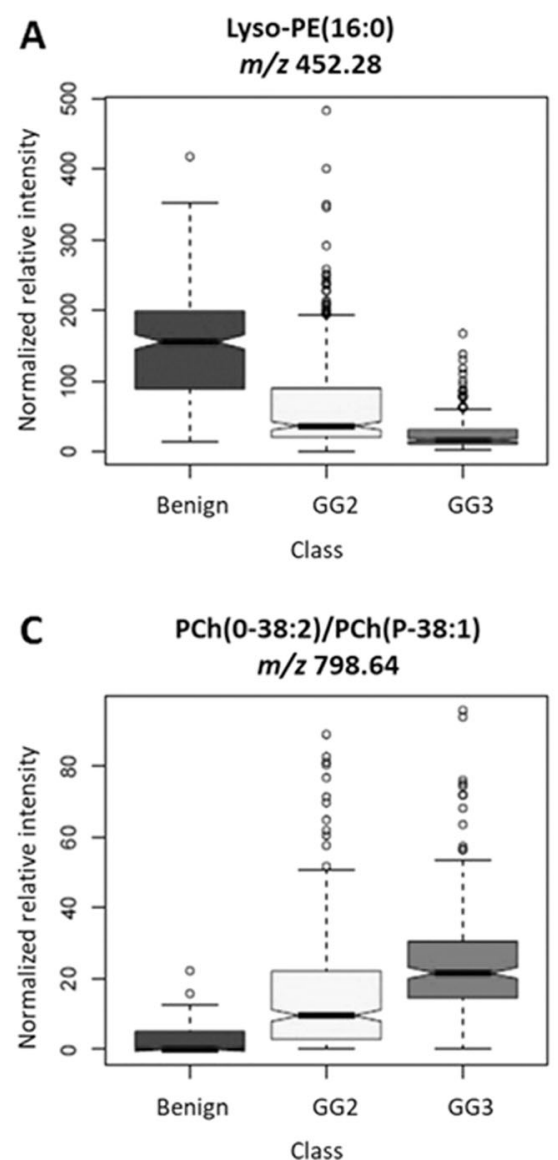

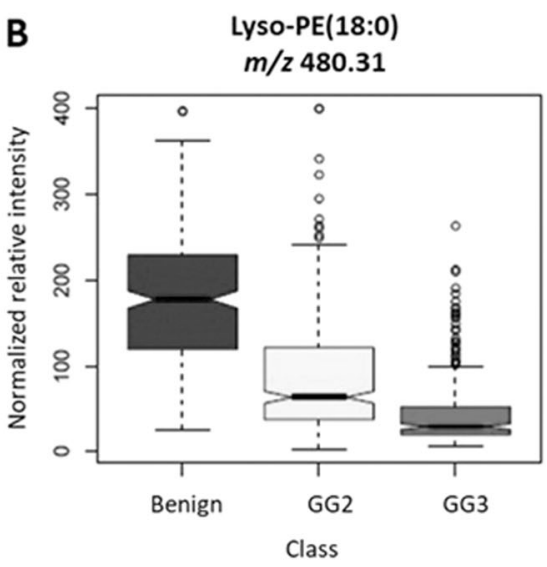

D
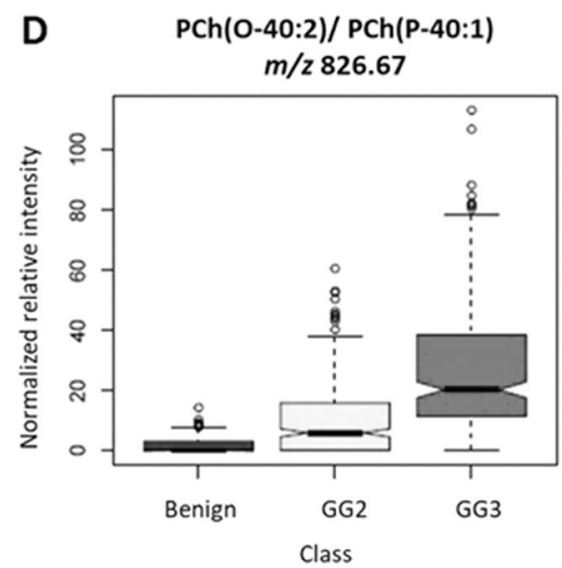

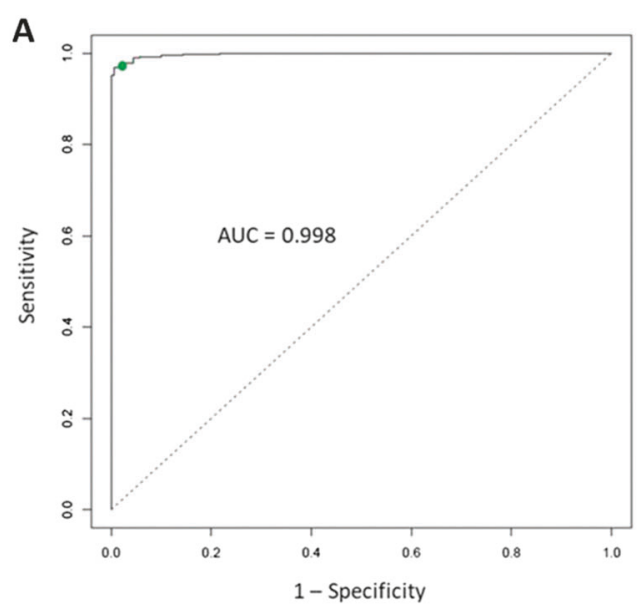

Fig. 4 Training and validation of an accurate metabolomic prostate cancer classifier. a The PCA/LDA model had excellent accuracy when used on the training cohort $(n=534)$ as displayed in the ROC curve. This model demonstrated an AUC of 0.994 on the training cohort. The green circle shows the selection of model threshold described in the text. Area under the curve (AUC), linear discriminant analysis (LDA), principal component analysis (PCA), receiver operating characteristic

offered a significant improvement in the validation cohort over the simpler univariate and logistic regression models. Therefore, we show here a validated cancer/benign classifier

B

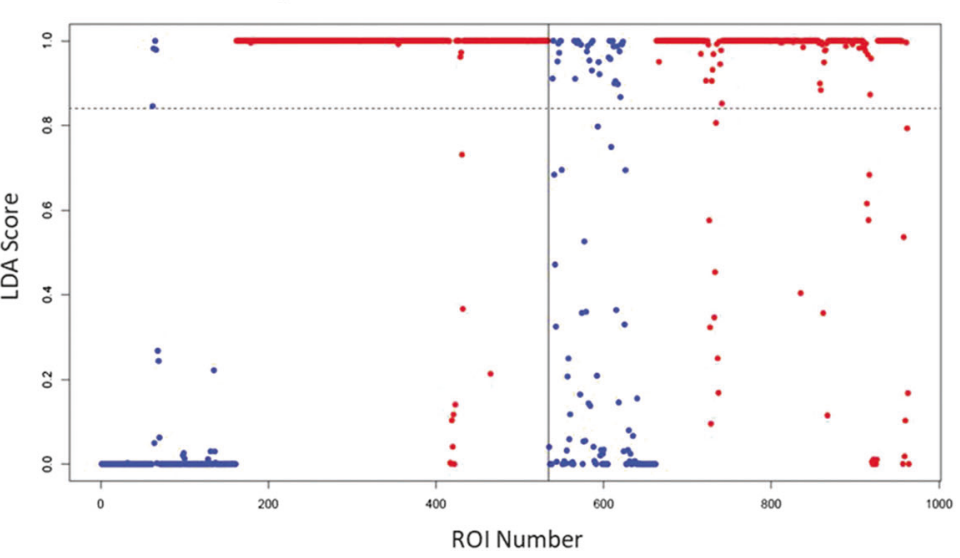

(ROC). b The PCA/LDA model had excellent accuracy when used on the training cohort $(n=534)$ as displayed in the ROC curve. This model demonstrated an AUC of 0.994 on the training cohort. The green circle shows the selection of model threshold described in the text. Area under the curve (AUC), linear discriminant analysis (LDA), principal component analysis (PCA), receiver operating characteristic (ROC)

with high statistical power that demonstrates very high sensitivity and balanced accuracies built on data obtained using needle biopsy cores. 
Table 2 Confusion matrix for the PCA/LDA model in training and validation cohorts

\begin{tabular}{llllll}
\hline Classified & \multicolumn{2}{l}{ Actual } & & \\
\cline { 2 - 3 } & \multicolumn{2}{l}{ Training cohort } & & \multicolumn{2}{l}{ Validation cohort } \\
\cline { 2 - 3 } \cline { 5 - 6 } & Benign & Cancer & & Benign & Cancer \\
\hline Benign & 157 & 10 & & 90 & 28 \\
Cancer & 4 & 363 & & 38 & 274 \\
Specificity (\%) & 97 & & & 70 & \\
Sensitivity (\%) & 97 & & & 91 & \\
Balanced accuracy (\%) & 97 & & & 85 & \\
\hline
\end{tabular}

\section{Discussion}

Identifying validated metabolomic biomarkers of prostate cancer can help characterize the biological processes that underlie cancer development and provide a biological basis for aggressive behavior. Using DESI-MSI we have identified metabolites with differential abundances between regions of benign and prostate cancer tissue including; citrate, lyso-PEs, glutamate, FAs, and phospholipids. Furthermore, despite challenges associated with standardizing this technology, we report DESI-MSI produces a metabolic classifier for prostate cancer that can be appropriately validated.

Previous studies using GC-MS and LC-MS techniques have identified metabolomic differences in patients with prostate cancer. One study identified increased levels of sarcosine in the urine of patients with PCa compared to healthy controls with further increases in patients whose cancer had metastasized [3]. In another study using plasma samples from $105 \mathrm{PCa}$ patients and 36 healthy controls, Zhou et al. [30] constructed a 15 metabolite model consisting of PE and PC species which demonstrated $94 \%$ sensitivity and $90 \%$ specificity, with metabolites that closely resembled those identified in this study. Finally, Zang et al. identified a panel of 40 metabolites from serum samples of 64 patients with PCa. This panel achieved $92 \%$ sensitivity and a 94\% specificity in distinguishing cancer patients from healthy controls. These metabolites consisted of FAs, amino acids, lysophospholipids, and bile acids and were linked to steroid hormone biosynthesis [31]. An important feature of these studies is that only the sarcosine study [3] validated its findings on a separate cohort of patients. Without such validation, biomarkers may represent coincidental associations between metabolites and cancer, and not be generally applicable to other patients. Even with validation, subsequent studies in other laboratories failed to confirm that sarcosine levels are reliably and significantly elevated in urine from prostate cancer patients (reviewed in ref. [32]). Perhaps chief among the challenges for sarcosine is that even when elevated, its levels are only slightly higher in cancer patients than in controls [3, 33-37]. In contrast, the current work demonstrates differential expression of individual metabolites that preferentially localize to prostate cancer cells (Fig. 2) and show clear separation between benign and cancer ROIs (Fig. 3) with $\log 2$ fold change between 1 and 10 (Table 1). While the results of the current study will require additional validation by other investigators, we view these factors as reasons for optimism.

To date, two studies have attempted to develop metabolic profiles for specific cancer grades with limited success. The first identified PChs, amino acids, arachidonic acid, and other FAs which yielded an AUC of 0.685 when comparing serum samples from 121 patients with low-grade cancers from the 85 patients with high-grade cancers [38]. While this result suggests that blood-based metabolomics may have clinical promise, most clinical decision making requires the ability to distinguish low grade from intermediate grade cancers [39]. Another study of 200 cases of prostate cancer and 200 controls found a positive correlation between energy and lipid metabolite levels and cancer aggressiveness [40]. Multiple FAs and glycerophospholipid species were increased in higher-grade cancers, along with $\alpha$-ketoglutarate and citrate [40]. Due to the difference in techniques used, results from previous studies cannot be directly compared since DESI-MSI cannot identify certain metabolites such as amino and bile acids; however, we observed the similar trend that FAs and lipids are more abundant in prostate cancer samples.

Differentially abundant metabolites identified in this study would be expected to provide the materials for prostate cancer growth and spread. We identified increased citrate levels in benign prostate tissue and increased glutamate in cancer tissue. Both metabolites reflect well-known energy alterations in prostate cancer [13]. Citrate is produced and stored in benign prostate epithelia for secretion into prostatic fluid. Prostate cancer cells eschew citrate storage, diverting it instead into the Krebs cycle to generate ATP and to be used as starting material for FA synthesis $[13,14]$. Increased abundance of glutamate in prostate cancer tissue provides further evidence that the Krebs cycle is operational. Glutamate is an intermediate within the glutamine pathway which normally functions to produce amino acids. Prostate cancer cells convert glutamate back into $\alpha$-ketoglutarate and subsequently to citrate, which is further utilized for FA biosynthesis. Prostate cancer cells incorporate these FAs into lipids that are incorporated into cell membranes and signaling molecules or catabolized for energy. Indeed, increased levels of glutamate have been correlated with more aggressive PCa [41].

Consistent with previous studies identifying increased FA biosynthesis in prostate cancer [16, 42-44], we 
identified elevated levels of two FAs, FA(20:2), FA(22:4), in prostate cancer tissue. In cancer, FAs regulate protein activation, act as a fuel source in the $\beta$-oxidation pathway and provide the building blocks for lipid production $[16,45]$. To coincide with increased FAs, there was also an increase in phospholipids in cancer tissue. Most of these species corresponded to PIs, PEs, and PChs which are components of cell membranes utilized for rapid cellular proliferation, and their differential abundance in cancer aligns with significantly higher rates of proliferation in cancer cells (Fig. 2). Increased biosynthesis of cell membrane lipids is facilitated by the upregulation of lipogenic enzymes such as SREBP1 and FASN which is commonly observed in prostate cancer [42, 44, 46]. Of the four metabolites that differentiated GG2 and GG3 cancers, two were PChs species. These two PChs were found at low levels in benign tissue, increased in prostate cancer, and further elevated with increased grade (Fig. 3). Prostate cancer cells are known to increase $\mathrm{PCh}$ production, to promote cell cycle progression [30, 47]. In contrast, insufficient levels of PCh species in G1 phase will induce apoptosis [48, 49]. Thus, increased FA and lipid biosynthesis appears to be critical features of prostate cancer growth.

Lipids in the $\mathrm{m} / \mathrm{z}$ range between 400 and 500 were highly enriched in benign prostate tissue. Aside from cholesterol sulfate, ions in this mass range have not been not reported in previous DESI-MSI studies. Using fragmentation analysis, we identified these ions as belonging to a family of lyso-PEs containing 16 and 18 carbon side chains (Table 1). In comparison to other anatomical zones of the prostate, lysophospholipids are selectively increased in benign tissue in the peripheral zone, which is the preferred site of $\mathrm{PCa}$ development [50]. While the mechanisms by which lysophospholipids might support prostate carcinogenesis have not been elucidated, various other cancer cells scavenge lysophospholipids, using them as fuel sources to support growth [51]. In the current study, lyso-PE(16:0) and lyso$\mathrm{PE}(18: 0)$ levels gradually decreased from benign to GG2 and were the lowest in GG3. This observation suggests that prostate cancer deplete lysophospholipids in proportion to their growth rates. Overall, the metabolites we found to be differentially abundant between benign and cancer tissue and those that vary with cancer grade reflect established altered metabolic pathways in PCa and highlight potential for the development and utilization of functional metabolomic biomarkers.

Interestingly, the metabolic profiles we observed in prostate cancer appear to reflect the grade of the case rather than local variation in cancer grade manifested at the level of the core. Of further interest, proliferation rates associated with GG of the case (Fig. 1), but not with GG of individual cores (Data not shown). These observations suggest that metabolic profiles are more sensitive to underlying clonal genetic and epigenetic influences in the tumor than they are to local influences. Additional studies will be needed to address this issue. Importantly, the work described here has identified metabolites that distinguished between GG2 and GG3. Although these two GGs were previously stratified into the same risk group regarding recommended treatment [52], they have very different survival outcomes and newer studies indicate that they should be treated differently. For example, a recent study showed that the risk of treatment failure rises from $20 \%$ of patients with GG2 cancer to $52 \%$ of patients with GG3 [25]. Because GG1 cases are considered non-life threatening, surgical treatment of low grade (GG1) cancers is no longer recommended, and therefore insufficient numbers of GG1 cases were available for analysis in this study. Future studies extend metabolomic analysis to GG1 biopsy tissue, which should yield additional insights into the metabolic landscape of indolent prostate cancer. Altogether, DESI-MSI has the potential to identify metabolites that distinguish prostate cancer GGs and in doing so, elucidate important biologic differences between indolent and aggressive prostate cancers.

In constructing a classifier, we investigated models with increasing levels of complexity. The univariate model demonstrated adequate AUCs which were increased when using the logistic regression model. Notably the logistic regression model had a good balanced accuracy on the training cohort but suffered a reduction on the test cohort. In contrast, the more complex PCA/LDA displayed superior balanced accuracies on both the training and test cohorts. LDA is an effective classifier that minimizes variability within classes while maximizing differences between classes. For these reasons, PCA/LDA was chosen as it has shown to be effective for classification models using metabolomics data [6]. The statistical workflow employed here to examine different modeling techniques ensured the logical selection of an accurate model while avoiding overfitting of the data.

Using the method described here, we were able to appreciate the tissue heterogeneity of the prostate needle biopsy core. The current study represents the smallest reported DESI-MSI pixel size employed on prostate tissue. In contrast to the two previous DESI-MSI prostate cancer reports that analyzed merged metabolite data representing $10 \mathrm{~mm}^{2}$ or more, the ROIs selected for analysis in the current study measured $<0.025 \mathrm{~mm}^{2}$ and typically contained four prostate glands (data not shown). Smaller ROIs allowed us to fully utilize the high-resolution DESI ion images to ensure that our cancer or benign ROIs were not confounded by other tissue types including; stroma, inflammation, or preneoplastic lesions. Additionally, numerous ROIs per sample increased the sample size of data points used for analysis, therefore significantly 
increasing statistical power and confidence in the resulting differential metabolites and model accuracies. Classification at sub-millimeter resolution is more representative to the true clinical need of identifying small regions of cancer intraoperatively and/or diagnostically. Ultimately, the model accuracies presented here are an improved indication on how DESI-MSI might perform in clinical applications.

DESI-MSI and related technologies have the potential to add new and important dimensions to routine and intraoperative diagnosis. DESI is non-destructive and therefore has the potential to be combined with advanced genomic profiling techniques and/or traditional H\&E and IHC staining. DESI requires no specialized analytic reagents (ex. antibodies or DNA primers), and involves no hybridization or amplification steps, therefore DESI-MSI can be performed much faster and cheaper than current profiling technologies. For example, a cancer and benign classification model might have limited impact in the pathology laboratory, where routine light microscopy sensitively and specifically identifies prostate cancer in tissue samples. However, this or a similar classifier has the potential to aid in the surgical setting. For example, metabolite profiles gathered using DESI-MSI can be used to train mass spectrometry devices that analyze electrocautery smoke intraoperatively to identify tissues in real time during surgery [53]. If successfully applied to prostate surgeries, metabolic profiles of prostate cancer such as the one reported here have the capacity to reduce the occurrence of positive surgical margins, which currently complicates $11-48 \%$ of prostate surgeries [54].

In summary, the results of this study further support the emergent field of cancer metabolomics for both biological and clinical purposes. Differentially abundant metabolites found in this study validate pre-established metabolic pathways highlighting an importance in altered FA and lipid biosynthesis in prostate cancer. Furthermore, this is the first DESI-MSI study on prostate cancer to fully utilize the spatial resolution of the images to increase statistical power and to appropriately build tissue heterogeneity into statistical models. Finally, this is the first study to create and validate a high-resolution metabolomic classifier. While the classifier is not yet sufficiently validated for clinical use, the ability to construct and validate a metabolic cancer classifier is an important step towards implementing this technology and metabolic profiling in prostate cancer diagnosis and surgical guidance.

Acknowledgements This project was funded by the South Eastern Ontario Medical Organization (SEAMO) (DMB) and the Britton Smith Chair in Surgery, Queen's University (to Dr. John Rudan). Nicole Morse is the recipient of a Canadian Institute of Healthcare Research Canadian Graduate Studies Master's (CIHR-CGS-M) award. We thank Dr. John F. Rudan and Dr. Seth Chitayat (Queen's University) for material and logistical support. We thank members of
Zoltan Takats' lab (Imperial College London) and Waters Corporation (Wilmslow UK) for advice, training, and technical support.

\section{Compliance with ethical standards}

Conflict of interest The authors declare that they have no conflict of interest.

Publisher's note: Springer Nature remains neutral with regard to jurisdictional claims in published maps and institutional affiliations.

\section{References}

1. Verma M, Banerjee HN. Metabolomic approaches in cancer epidemiology. Diseases. 2015;3:167-75.

2. Priolo C, Pyne S, Rose J, Regan ER, Zadra G, Photopoulos C, et al. AKT1 and MYC induce distinctive metabolic fingerprints in human prostate cancer. Cancer Res. 2014;74:7198-204.

3. Sreekumar A, Poisson LM, Rajendiran TM, Khan AP, Cao Q, $\mathrm{Yu}$ J, et al. Metabolomic profiles delineate potential role for sarcosine in prostate cancer progression. Nature. 2009;457:910-4.

4. Snyder NW, Mesaros C, Blair IA. Translational metabolomics in cancer research. Biomark Med. 2015;9:821-34.

5. Eberlin LS, Ferreira CR, Dill AL, Ifa DR, Cooks RG. Desorption electrospray ionization mass spectrometry for lipid characterization and biological tissue imaging. Biochim Biophys Acta. 2011; 1811:946-60.

6. Veselkov KA, Mirnezami R, Strittmatter N, Goldin RD, Kinross J, Speller AV, et al. Chemo-informatic strategy for imaging mass spectrometry-based hyperspectral profiling of lipid signatures in colorectal cancer. Proc Natl Acad Sci USA. 2014;111:1216-21.

7. Kelloff GJ, Choyke P, Coffey DS. Prostate Cancer Imaging Working G. Challenges in clinical prostate cancer: role of imaging. AJR Am J Roentgenol. 2009;192:1455-70.

8. Fleming M, Ravula S, Tatishchev SF, Wang HL. Colorectal carcinoma: pathologic aspects. J Gastrointest Oncol. 2012;3:153-73.

9. Cancer Genome Atlas Research Network. The molecular taxonomy of primary prostate. Cancer Cell. 2015;163:1011-25.

10. Epstein JI, Egevad L, Amin MB, Delahunt B, Srigley JR, Humphrey PA, et al. The 2014 International Society of Urological Pathology (ISUP) consensus conference on gleason grading of prostatic carcinoma: definition of grading patterns and proposal for a new grading system. Am J Surg Pathol. 2016;40:244-52.

11. Narayan VM, Konety BR, Warlick C. Novel biomarkers for prostate cancer: an evidence-based review for use in clinical practice. Int J Urol. 2017;24:352-60.

12. Warburg O. On the origin of cancer cells. Science. 1956;123: 309-14.

13. Costello LC, Franklin RB. The intermediary metabolism of the prostate: a key to understanding the pathogenesis and progression of prostate malignancy. Oncology. 2000;59:269-82.

14. Costello LC, Liu Y, Franklin RB, Kennedy MC. Zinc inhibition of mitochondrial aconitase and its importance in citrate metabolism of prostate epithelial cells. J Biol Chem. 1997;272:28875-81.

15. Wu X, Daniels G, Lee P, Monaco ME. Lipid metabolism in prostate cancer. Am J Clin Exp Urol. 2014;2:111-20.

16. Zadra G, Photopoulos C, Loda M. The fat side of prostate cancer. Biochim Biophys Acta. 2013;1831:1518-32.

17. Kerian KS, Jarmusch AK, Pirro V, Koch MO, Masterson TA, Cheng L, et al. Differentiation of prostate cancer from normal tissue in radical prostatectomy specimens by desorption electrospray ionization and touch spray ionization mass spectrometry. Analyst. 2015;140:1090-8. 
18. Banerjee S, Zare RN, Tibshirani RJ, Kunder CA, Nolley R, Fan R, et al. Diagnosis of prostate cancer by desorption electrospray ionization mass spectrometric imaging of small metabolites and lipids. Proc Natl Acad Sci USA. 2017;114:3334-9.

19. Guijas C, Montenegro-Burke JR, Domingo-Almenara X, Palermo A, Warth B, Hermann G, et al. METLIN: a technology platform for identifying knowns and unknowns. Anal Chem. 2018;90:3156-64.

20. Zhao Z, Xu Y. An extremely simple method for extraction of lysophospholipids and phospholipids from blood samples. J Lipid Res. 2010;51:652-9.

21. Bligh EG, Dyer WJ. A rapid method of total lipid extraction and purification. Can J Biochem Physiol. 1959;37:911-7.

22. Kind T, Liu KH, Lee DY, DeFelice B, Meissen JK, Fiehn O. LipidBlast in silico tandem mass spectrometry database for lipid identification. Nat Methods. 2013;10:755-8.

23. Benjamini Y, Drai D, Elmer G, Kafkafi N, Golani I. Controlling the false discovery rate in behavior genetics research. Behav Brain Res. 2001;125:279-84.

24. Berman DM, Epstein JI. When is prostate cancer really cancer? Urol Clin North Am. 2014;41:339-46.

25. Offermann A, Hohensteiner S, Kuempers C, Ribbat-Idel J, Schneider F, Becker F, et al. Prognostic value of the new prostate Cancer International Society of urological pathology grade groups. Front Med. 2017;4:157.

26. Balog J, Sasi-Szabo L, Kinross J, Lewis MR, Muirhead LJ, Veselkov $\mathrm{K}$, et al. Intraoperative tissue identification using rapid evaporative ionization mass spectrometry. Sci Transl Med. 2013;5:194ra93.

27. Bryan K, Brennan L, Cunningham P. MetaFIND: a feature analysis tool for metabolomics data. BMC Bioinform. 2008;9:470.

28. Gerbig S, Golf O, Balog J, Denes J, Baranyai Z, Zarand A, et al. Analysis of colorectal adenocarcinoma tissue by desorption electrospray ionization mass spectrometric imaging. Anal Bioanal Chem. 2012;403:2315-25.

29. Schafer KC, Balog J, Szaniszlo T, Szalay D, Mezey G, Denes J, et al. Real time analysis of brain tissue by direct combination of ultrasonic surgical aspiration and sonic spray mass spectrometry. Anal Chem. 2011;83:7729-35.

30. Zhou X, Mao J, Ai J, Deng Y, Roth MR, Pound C, et al. Identification of plasma lipid biomarkers for prostate cancer by lipidomics and bioinformatics. PLoS ONE. 2012;7:e48889.

31. Zang X, Jones CM, Long TQ, Monge ME, Zhou M, Walker LD, et al. Feasibility of detecting prostate cancer by ultraperformance liquid chromatography-mass spectrometry serum metabolomics. J Proteome Res. 2014;13:3444-54.

32. Issaq HJ, Veenstra TD. Is sarcosine a biomarker for prostate cancer? J Sep Sci. 2011;34:3619-21.

33. Jentzmik F, Stephan C, Miller K, Schrader M, Erbersdobler A, Kristiansen $G$, et al. Sarcosine in urine after digital rectal examination fails as a marker in prostate cancer detection and identification of aggressive tumours. Eur Urol. 2010;58:12-8. discussion20-1.

34. Colleselli D, Stenzl A, Schwentner C, et al. Re: Florian Jentzmik, Carsten Stephan, Kurt Miller, et al. Sarcosine in urine after digital rectal examination fails as a marker in prostate cancer detection and identification of aggressive tumours. Eur Urol 2010;58:12-8. Eur Urol. 2010;58:e51.

35. Struys EA, Heijboer AC, van Moorselaar J, Jakobs C, Blankenstein MA. Serum sarcosine is not a marker for prostate cancer. Ann Clin Biochem. 2010;47:282.

36. Jentzmik F, Stephan C, Lein M, Miller K, Kamlage B, Bethan B, et al. Sarcosine in prostate cancer tissue is not a differential metabolite for prostate cancer aggressiveness and biochemical progression. J Urol. 2011;185:706-11.

37. Cao DL, Ye DW, Zhu Y, Zhang HL, Wang YX, Yao XD. Efforts to resolve the contradictions in early diagnosis of prostate cancer: a comparison of different algorithms of sarcosine in urine. Prostate Cancer Prostatic Dis. 2011;14:166-72.

38. Osl M, Dreiseitl S, Pfeifer B, Weinberger $\mathrm{K}$, Klocker $\mathrm{H}$, Bartsch G, et al. A new rule-based algorithm for identifying metabolic markers in prostate cancer using tandem mass spectrometry. Bioinformatics. 2008;24:2908-14.

39. Alam R, Carter HB, Epstein JI, Tosoian JJ. Active surveillance of prostate cancer: current state of practice and utility of multiparametric magnetic resonance imaging. Rev Urol. 2017;19:77-88.

40. Mondul AM, Moore SC, Weinstein SJ, Karoly ED, Sampson JN, Albanes D. Metabolomic analysis of prostate cancer risk in a prospective cohort: the alpha-tocolpherol, beta-carotene cancer prevention (ATBC) study. Int J Cancer. 2015;137:2124-32.

41. Koochekpour S, Majumdar S, Azabdaftari G, Attwood K, Scioneaux R, Subramani D, et al. Serum glutamate levels correlate with Gleason score and glutamate blockade decreases proliferation, migration, and invasion and induces apoptosis in prostate cancer cells. Clin Cancer Res. 2012;18:5888-901.

42. Huang WC, Li X, Liu J, Lin J, Chung LW. Activation of androgen receptor, lipogenesis, and oxidative stress converged by SREBP-1 is responsible for regulating growth and progression of prostate cancer cells. Mol Cancer Res. 2012;10:133-42.

43. Madigan AA, Rycyna KJ, Parwani AV, Datiri YJ, Basudan AM, Sobek KM, et al. Novel nuclear localization of fatty acid synthase correlates with prostate cancer aggressiveness. Am J Pathol. 2014; 184:2156-62.

44. Shurbaji MS, Kalbfleisch JH, Thurmond TS. Immunohistochemical detection of a fatty acid synthase (OA-519) as a predictor of progression of prostate cancer. Hum Pathol. 1996;27:917-21.

45. Liu Y. Fatty acid oxidation is a dominant bioenergetic pathway in prostate cancer. Prostate Cancer Prostatic Dis. 2006;9:230-4.

46. Yoshii Y, Furukawa T, Oyama N, Hasegawa Y, Kiyono Y, Nishii R, et al. Fatty acid synthase is a key target in multiple essential tumor functions of prostate cancer: uptake of radiolabeled acetate as a predictor of the targeted therapy outcome. PLoS ONE. 2013;8:e64570.

47. Duscharla D, Bhumireddy SR, Lakshetti S, Pospisil H, Murthy $\mathrm{PV}$, Walther R, et al. Prostate cancer associated lipid signatures in serum studied by ESI-tandem mass spectrometryas potential new biomarkers. PLoS ONE. 2016;11:e0150253.

48. Cui Z, Houweling M, Chen MH, Record M, Chap H, Vance DE, et al. A genetic defect in phosphatidylcholine biosynthesis triggers apoptosis in Chinese hamster ovary cells. J Biol Chem. 1996;271: 14668-71.

49. Ridgway ND. The role of phosphatidylcholine and choline metabolites to cell proliferation and survival. Crit Rev Biochem Mol Biol. 2013;48:20-38.

50. Al Kadhi O, Traka MH, Melchini A, Troncoso-Rey P, Jurkowski $\mathrm{W}$, Defernez M, et al. Increased transcriptional and metabolic capacity for lipid metabolism in the peripheral zone of the prostate may underpin its increased susceptibility to cancer. Oncotarget. 2017;8:84902-16.

51. Kamphorst JJ, Cross JR, Fan J, de Stanchina E, Mathew R, White EP, et al. Hypoxic and Ras-transformed cells support growth by scavenging unsaturated fatty acids from lysophospholipids. Proc Natl Acad Sci USA. 2013;110:8882-7.

52. Rodrigues G, Warde P, Pickles T, Crook J, Brundage M, Souhami $\mathrm{L}$, et al. Pre-treatment risk stratification of prostate cancer patients: a critical review. Can Urol Assoc J. 2012;6:121-7.

53. Golf O, Muirhead LJ, Speller A, Balog J, Abbassi-Ghadi N, Kumar S, et al. XMS: cross-platform normalization method for multimodal mass spectrometric tissue profiling. J Am Soc Mass Spectrom. 2015;26:44-54.

54. Silberstein JL, Eastham JA. Significance and management of positive surgical margins at the time of radical prostatectomy. Indian J Urol. 2014;30:423-8. 